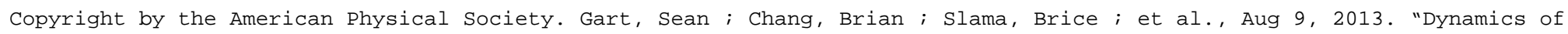
squeezing fluids: Clapping wet hands," PHYSICAL REVIEW E 88(2): 023007. DOI: 10.1103/PhysRevE.88.023007.

PHYSICAL REVIEW E 88, 023007 (2013)

\title{
Dynamics of squeezing fluids: Clapping wet hands
}

\author{
Sean Gart, ${ }^{1}$ Brian Chang, ${ }^{1}$ Brice Slama, ${ }^{2}$ Randy Goodnight, ${ }^{1}$ Soong Ho Um, ${ }^{3}$ and Sunghwan Jung ${ }^{1, *}$ \\ ${ }^{1}$ Department of Engineering Science and Mechanics, Virginia Tech, Blacksburg, Virginia 24061 \\ ${ }^{2}$ Department of Mechanics, Ecole Polytechnique, 91128 Palaiseau, Cedex \\ ${ }^{3}$ School of Chemical Engineering and SKKU Advanced Institute of Nanotechnology (SAINT), \\ Sungkyunkwan University, Gyeonggi-do, Republic of Korea
}

(Received 30 November 2012; revised manuscript received 26 February 2013; published 9 August 2013)

\begin{abstract}
Droplets splash around when a fluid volume is quickly compressed. This phenomenon has been observed during common activities such as kids clapping with wet hands. The underlying mechanism involves a fluid volume being compressed vertically between two objects. This compression causes the fluid volume to be ejected radially and thereby generate fluid threads and droplets at a high speed. In this study, we designed and performed laboratory experiments to observe the process of thread and drop formation after a fluid is squeezed. A thicker rim at the outer edge forms and moves after the squeezing, and then becomes unstable and breaks into smaller drops. This process differs from previous well-known examples (i.e., transient crown splashes and continuous water bells) in aspects of transient fluid feeding, expanding rim dynamics, or sparsely distributed drops. We compared experimental measurements with theoretical models over three different stages; early squeezing, intermediate sheet-expansion, and later break-up of the liquid thread. In the earlier stage, the fluid is squeezed and its initial velocity is governed by the lubrication force. The outer rim of the liquid sheet forms curved trajectories due to gravity, inertia, drag, and surface tension. At the late stage, drop spacing set by the initial capillary instability does not change in the course of rim expansion, consequently final ejected droplets are very sparse compared to the size of the rim.
\end{abstract}

DOI: 10.1103/PhysRevE.88.023007

PACS number(s): 47.20.Dr, 47.55.D-, 47.55.nm

\section{INTRODUCTION}

The atomization process of a liquid thread is observed in many industrial applications (e.g., coating [1], cooling, etc. [2,3]) and everyday life (e.g., raindrop formation [4], clapping wet hands). In particular, we have all experienced the splashing effects of clapping with wet hands. When water splashes, numerous water droplets, rather than fluid threads, are dispersed. Such a squeezing motion of the hands makes the fluid in between eject and eventually break into drops. This outburst of fluid motion is the primary motivation behind this study.

Drop formation from a fluid sheet has been observed and studied in two notable cases: a crown splash [5-10] and a water bell [11-15]. In a crown splash, the crown shape of the fluid results from an instability along a cylindrical fluid-sheet forming due to transient fluid impact. In this process, a drop of a fluid is released and impacts a bath of the same fluid at rest or a solid substrate. After impact, a cylindrical sheet forms and moves upwards, eventually creating droplets along the edge of the fluid rim [16]. Liquid crowns of various geometries have been studied extensively; on a bulk of the same fluid $[10,17]$, on a solid wall $[18,19]$, on a thin fluid layer $[20,21]$, on a rod [22,23], and more. The water bell $[14,15]$ is another example sharing some features with our study of clapping with wet hands, i.e., the fluid rim connected to the sheet. When a continuous fluid jet hits a localized solid obstacle (i.e., a rod), the jet radially spreads and forms a circular sheet called a water bell [24]. Similar to the liquid crown, the water bell has the drop-formation mechanism in which the fluid sheet radially expands and breaks into smaller droplets.

\footnotetext{
*sunnyjsh@vt.edu
}

Uniqueness of the proposed work, clapping wet hands, lies in these three points: First, the flow of fluid to a liquid sheet is transient and follows the inverse power law due to a lubricating flow between two plates, which is not observed in the other two cases. Second, the thick rim in this case expands radially in a different manner than other liquid sheets. All water bells are steadily forming both a rim and a liquid sheet without temporal dynamics due to a continuous liquid feed. Third, an undulatory thick rim is developed at an early stage and later gravity and radial expansion amplifies this rim's waveness. But, a crown splash develops a decelerating liquid sheet against gravity and becomes unstable at a later stage when it reaches close to its maximum position. Water bells are either closing the fluid sheet at the lower end or splashing radially with a continuous jet.

In this paper, we investigate how a fluid sheet and rim develop and move due to the squeezing motion of disks, and how fluid droplets form from the rim due to the capillary instability as illustrated in Fig. 1. Again, this study is motivated by the familiar act of clapping with wet hands, which has unique features compared to traditional splash problems, e.g., crown splashes and water bells. First, Sec. II describes the experimental setup and procedure. In Sec. III, we compare experimental data with three theoretical models: the lubrication flow, the ejecting dynamics, and the capillary instability of the rim. Finally, we discuss the conclusion of our findings and the future direction in Sec. IV.

\section{EXPERIMENTAL METHODS}

The experimental setup is designed to understand and mimic the motion of clapping hands: a squeezing fluid motion caused by two circular disks [see Fig. 2(a)]. An upper disk is actuated to fall vertically at a fixed speed and eventually 


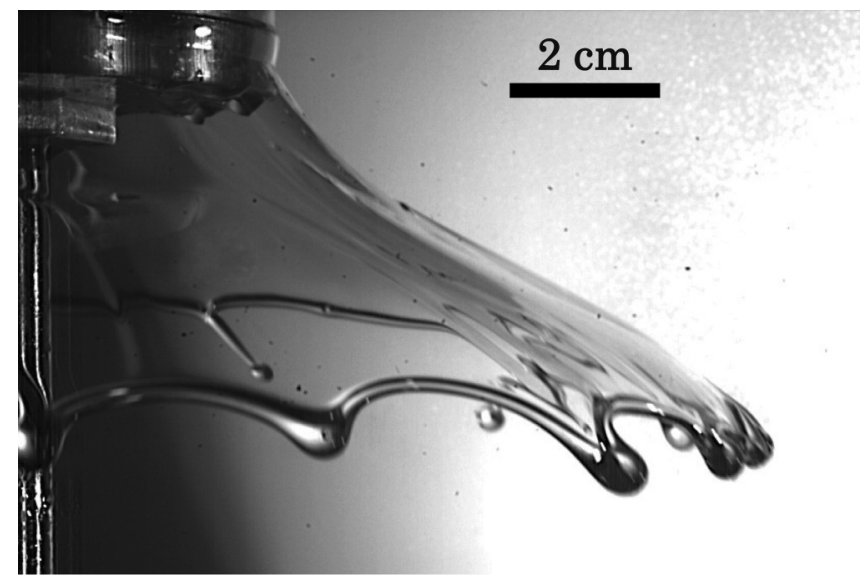

FIG. 1. Image of a liquid sheet (silicone oil of $100 \mathrm{cSt}$ ) squeezed from two clapping plates at the velocity of $10.2 \mathrm{~cm} / \mathrm{s}$.

collides with a bottom disk. Both disks have the same radius $\left(R_{\text {disk }}=3.8 \mathrm{~cm}\right)$ and are aligned accurately in order to avoid any sliding motion. The impact speed $\left(V_{\text {disk }}\right)$ of the upper disk varies from 5 to $13 \mathrm{~cm} / \mathrm{s}$ and is controlled by a linear actuator (Xslide XN10-0040-E04-71, Velmex Co.). The fluid motion is recorded at $1000 \mathrm{~Hz}$ by using either one MotionXtra N3 from the side or two Fastcam APX RS Photrons from

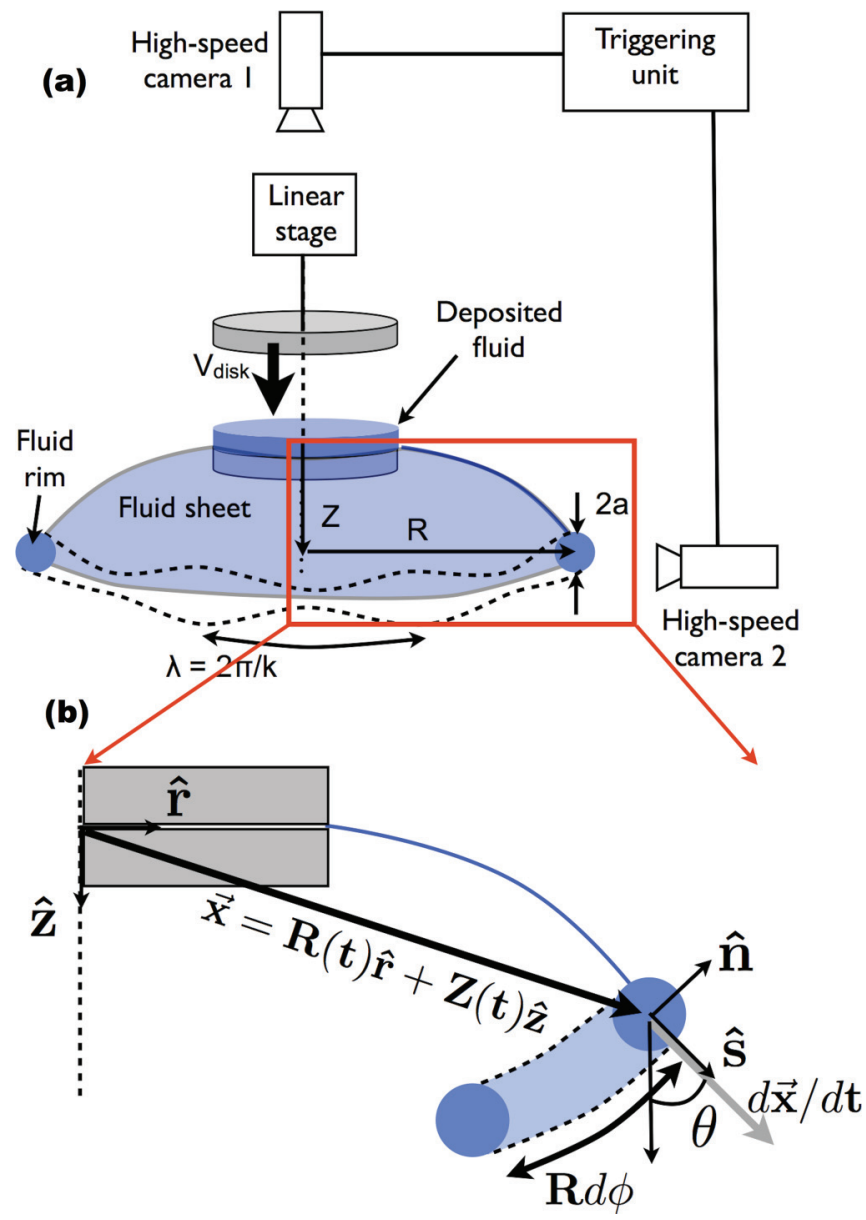

FIG. 2. (Color online) (a) Experimental apparatus and schematics of the sheet of fluid being ejected from the plates. (b) Zoomed schematics to explain the coordinate system of the rim dynamics. both the top and the side. The high-speed camera on the top is located approximately $2 \mathrm{~m}$ away from the disk to prevent any optical distortion. We experimented using silicon oil (Clearco Products) with kinematic viscosities $(v=\mu / \rho$; where $\mu$ is absolute fluid viscosity and $\rho$ is fluid density) of 20,50 , and $100 \mathrm{cSt}$ and surface tensions $(\gamma)$ of 20.6, 20.8, and 20.9 dynes/cm, respectively. We also used a mixture of $80 \%$ glycerol and $20 \%$ water of about $75 \mathrm{cSt}$ to test a high surface tension fluid of 65.7 dynes/cm [25]. For each experiment, the same amount of fluid $(7 \mathrm{ml})$ is first degassed and gently deposited on the top of the lower disk using a syringe to remove any initial bubbles in the bulk. The thin fluid layer deposited on the lower disk is about $1.5 \mathrm{~mm}$ in height.

The dimensionless parameters for describing the clapping hands experiment are the Reynolds number, $\operatorname{Re}=V_{\text {disk }} R_{\text {disk }} / \nu$; the Weber number, We $=\rho V_{\text {disk }}^{2} R_{\text {disk }} / \gamma$; and the Ohnesorge number, $\mathrm{Oh}=\mu / \sqrt{\rho \gamma R_{\text {disk }}}=\sqrt{\mathrm{We}} /$ Re. Our experiments cover ranges of $\mathrm{Oh}=0.02 \sim 0.1, \mathrm{Re}=20 \sim 200$, and $\mathrm{We}=2 \sim 20$.

When the two plates squeeze deposited fluids within about 10 to $30 \mathrm{~ms}$, the fluid is ejected in a mostly radial direction through the small gap between the two plates. In the course of squeezing, a fluid rim is quickly formed in about $20 \mathrm{~ms}$ (capillary timescale; $t_{\text {capillary }}=\sqrt{\rho a^{3} / \gamma}$ ) on the edge of the sheet primarily due to surface tension. The measured rim radius (a) is about $2 \mathrm{~mm}$, is independent of the disk speed, and close to the capillary length $(\sqrt{\gamma / \rho g} \approx 1.5 \mathrm{~mm})$. This indicates that the formation of the rim is mainly due to the capillary force and happens quickly in about $20 \mathrm{~ms}$. In the intermediate stage, the rim and sheet curve down due to gravity and decelerate due to drag and surface tension [see images after $t=40 \mathrm{~ms}$ in Fig. 3(a)]. Eventually, an instability will be initiated along the rim and drops will form on the tip of the fluid rim as shown in the images at $t=0,40,80,120,160 \mathrm{~ms}$ in Fig. 3(a). Depending on fluid viscosities and clapping speeds, variations in the fluid sheet and rim are observed, as shown in Figs. 3(b)-3(d).

Recorded images from the two high-speed cameras are analyzed using the MATLAB image processing toolbox to determine the position, size, and wavelength of the fluid rim. This program analyzes the intensity of each rim radius; the radius is found by locating the largest peak in intensity and the location of this peak will be the distance between the rim and the disk center. Then, the program analyzes the luminosity around the rim versus the angle for a certain range of angles as the pictures are difficult to analyze on the whole semi-arc. These experimental results from image analysis will be discussed and compared with theoretical models in the next section.

\section{RESULT}

\section{A. Squeezing flow: Early stage}

When the upper plate strikes the thin fluid on the lower plate, the fluid splashes radially due to the squeezing motion of the two plates. Due to the small thickness of the fluid $(\sim 1.5 \mathrm{~mm})$ and high fluid viscosity $(20 \sim 100 \mathrm{cSt})$, the low-Reynolds number lubrication approximation [26] can be considered in our experiments. The impact force $\left(F_{0}\right)$ from the upper disk is presumably proportional to the impact 


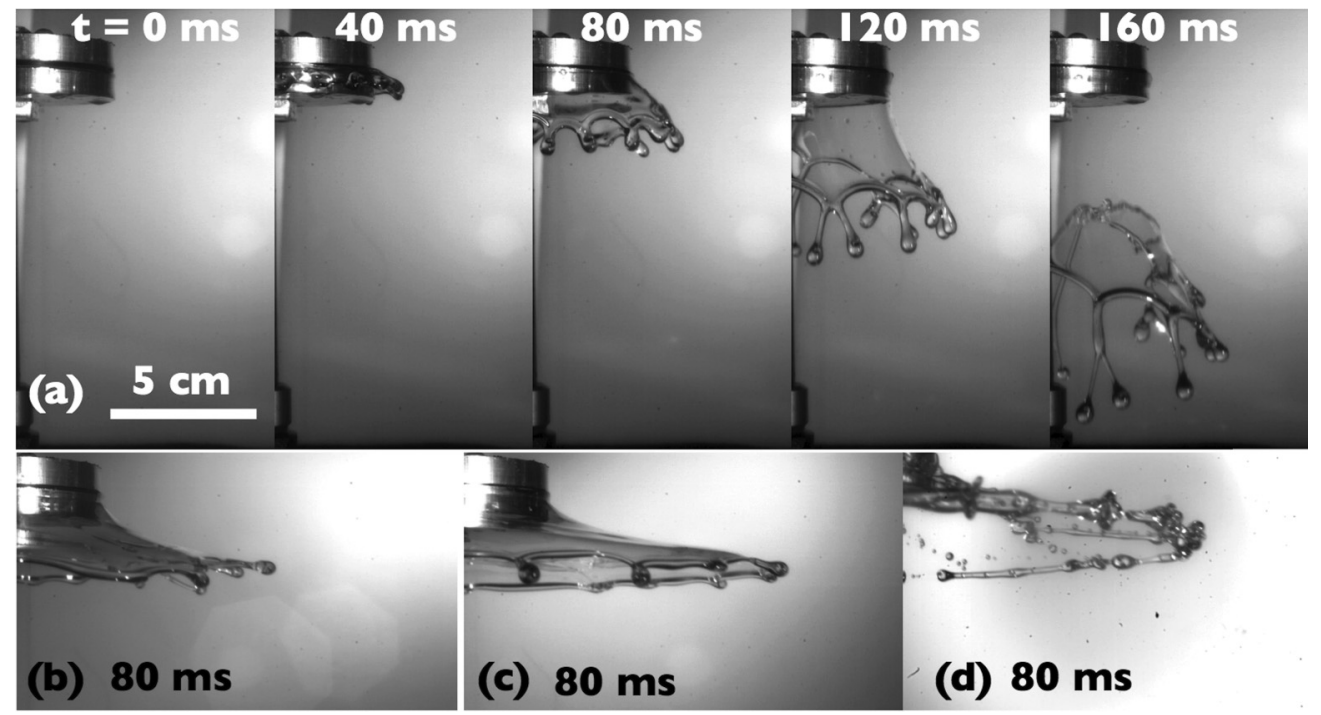

FIG. 3. Side-viewed image sequences (a) at $t=0,40,80,120$, and $160 \mathrm{~ms}$ with viscosity of $100 \mathrm{cSt}$ and $V_{\text {disk }}=5.08 \mathrm{~cm} / \mathrm{s}$, at $t=80 \mathrm{~ms}$ with $V_{\text {disk }}=12.70 \mathrm{~cm} / \mathrm{s}$ with viscosity of (b) $100 \mathrm{cSt}$, (c) $50 \mathrm{cSt}$, and (d) at $t=80 \mathrm{~ms}$ with $V_{\text {disk }}=12.70 \mathrm{~cm} / \mathrm{s} \mathrm{with} 75 \mathrm{cSt}$ water-glycerol mixture.

plate velocity $\left(V_{\text {disk }}\right)$ [27] and is then applied to compress the fluid in between. We can assume that the total fluid volume is conserved $\left(R(t)^{2} H(t)=R_{\text {disk }}^{2} H_{0}\right)$ and then solve the lubrication equation with initial conditions $\left(\left.H\right|_{t=0}=H_{0}\right.$, $\left.\left.R\right|_{t=0}=R_{\text {disk }}\right)$. The continuity equation in terms of radial and vertical velocities $\left(v_{r}, v_{z}\right)$ and the nontrivial component $(r)$ of the momentum in a cylindrical coordinate are written as

$$
\frac{1}{r} \frac{\partial}{\partial r}\left(r v_{r}\right)+\frac{\partial}{\partial z} v_{z}=0, \quad \frac{\partial P}{\partial r}=\mu \frac{\partial v_{r}}{\partial z^{2}} .
$$

If one solves for the vertical velocity, then $P(r)$ yields

$$
P(r)=-3 \mu \frac{r^{2}}{H^{3}} \frac{\partial H}{\partial t} .
$$

Integrating the pressure $P(r)$ over the plate surface $(r \in$ $\left[0, R_{\text {disk }}\right]$ and $\left.\theta \in[0,2 \pi]\right)$ gives the force exerted by the plate:

$$
F_{0}=\frac{3 \pi \mu}{2} \frac{R(t)^{4}}{H(t)^{3}} \frac{\partial H}{\partial t} .
$$

Finally, using the constant volume condition $[R(t)=$ $\left.R_{\text {disk }} \sqrt{H_{0} / H(t)}\right]$ gives the fluid height

$$
H(t)=H_{0}\left(1+\frac{8}{3 \pi \mu} \frac{H_{0}^{2} F_{0}}{R_{\mathrm{disk}}^{4}} t\right)^{-1 / 4} .
$$

For reference, this height expression is different from other lubrication calculations using the constant contact area condition [28]. Combining the linear relation of impact force with disk speed [27], the initial radial velocity will be

$$
\left.\frac{d R}{d t}\right|_{t=0}=\frac{H_{0}^{2} F_{0}}{3 \pi \rho \nu R_{\mathrm{disk}}^{3}} \propto V_{\mathrm{disk}} \frac{\nu_{\mathrm{water}}}{\nu} .
$$

It indicates that the initial radial velocity of the ejecting fluid is proportional to the clapping speed and inversely proportional to the fluid viscosity, which is in good agreement with the experimental observations with $R^{2}=0.82$ as shown in Fig. 4 .

\section{B. Expanding Rim: Intermediate stage}

One of the fascinating features in this experiment is the spreading of a thick fluid rim caused by the collision of two plates. The static shape of a closed fluid sheet, like a flowing fluid balloon, has been studied in Refs. [15,24]. Here, we will develop our model for the rim dynamics by considering various effects on a fluid rim in a coordinate system described in Fig. 2(b).

The mass of a small section of $\operatorname{rim} \rho \pi a^{2} R d \phi$ is moving in a curved trajectory where $\phi$ is the azimuthal angle in a cylindrical coordinate. Hence, the centrifugal force acts outwards as

$$
\vec{F}_{c}=\rho \pi a^{2} R d \phi \frac{|d \vec{x} / d t|^{2}}{R_{c}} \hat{n},
$$

where $\vec{x}(=R \hat{r}+Z \hat{z})$ is the position vector of a rim, $\hat{n}$ is the unit vector normal to the rim's trajectory, $R_{c}=(-d \theta / d s)^{-1}$ is the radius of curvature of the rim's trajectory, and the drag

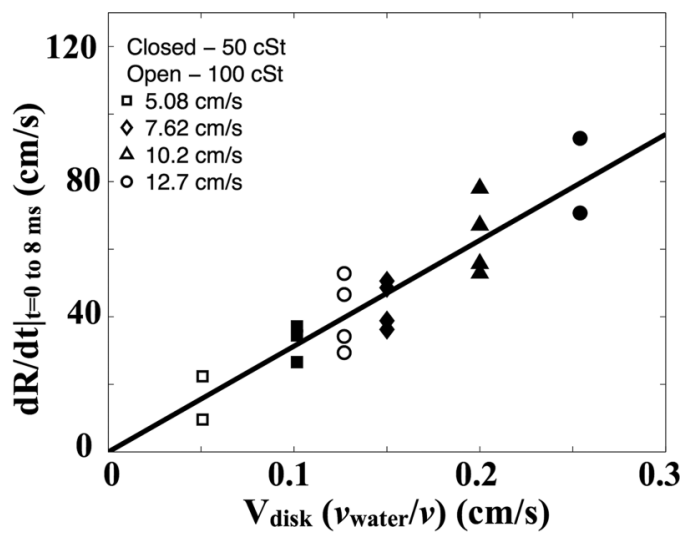

FIG. 4. Initial radial velocity of the rim vs. normalized disk velocity with different viscosities. The solid line is the best fit line with $R^{2}=0.82$. Initial radial velocity is measured over an 8-ms interval just after the clapping. 
force acts opposite to the rim's trajectory as

$$
\vec{F}_{d}=-\frac{1}{2} \rho C_{D}|d \vec{x} / d t|^{2}(2 a R d \phi) \hat{s},
$$

where $\hat{s}$ is the tangential unit vector along the rim's trajectory, $R$ is the radial distance of the rim, and $C_{D}$ is the drag coefficient. There are different models of the drag coefficient describing drag on a drop or a liquid film [29], but we have chosen $24 / \mathrm{Re}_{\text {air }}$, using air kinematic viscosity for simplicity. Since a thin fluid sheet is connected to the back side of the rim, the tangential capillary force can be expressed as

$$
\vec{F}_{\gamma 1}=-2 \gamma R d \phi \hat{s},
$$

where the factor of 2 is due to the upper and lower sides of a liquid sheet. Also, the capillary force acts along the cylinder's surface due to the curved cylindrical rim. The resultant force points in the negative normal direction as

$$
\vec{F}_{\gamma 2}=-\gamma a \frac{R}{R_{a}} d \phi \hat{n},
$$

where $R_{a}=R / \cos \theta$ is the azimuthal curvature of the rim. Last, gravity pulls the rim downward as

$$
\vec{F}_{g}=\rho g \pi a^{2} R d \phi \hat{z} .
$$

After adding all above forces, we write the governing equation for the liquid rim as

$$
\rho \pi a^{2} R d \phi \frac{d^{2} \vec{x}}{d t^{2}}=\vec{F}_{c}+\vec{F}_{d}+\vec{F}_{\gamma 1}+\vec{F}_{\gamma 2}+\vec{F}_{g},
$$

Then, we can solve this equation numerically using the following boundary conditions: $\left.R\right|_{t=0}=$ $R_{\text {disk }},\left.z\right|_{t=0}, d z /\left.d t\right|_{t=0}=0$, and $d R /\left.d t\right|_{t=0}$ is from the mean radial velocity in Fig. 4 at a given fluid viscosity and clapping speed as a result of lubrication force, which is described in the previous section.

Simulation results are compared with experiments in Fig. 5. Here, trajectories of only gravity (open squares), the full model solving Eq. (11) (open circles), and experiments (closed circles) exhibit a similar parabolic profile. In experiments, the position of the end tip of the silicone oil sheet is tracked instead

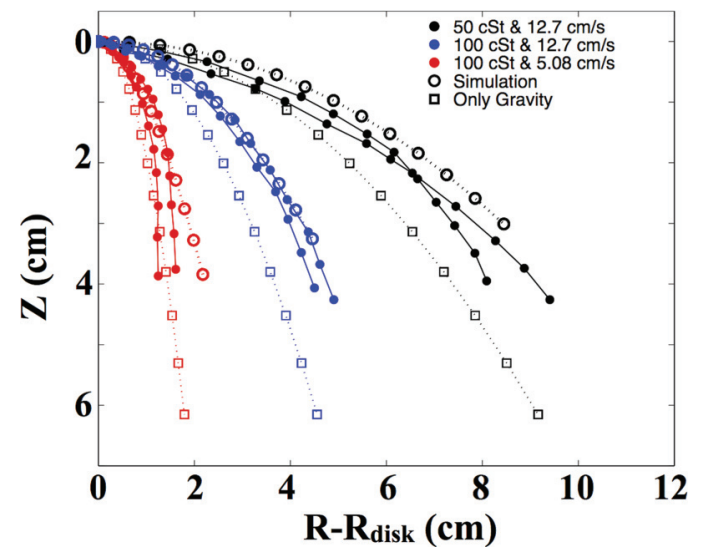

FIG. 5. (Color online) Positions of fluid rims with different velocities and viscosities. Open symbols are from experiments and closed symbols are from numerically solving Eq. (11). Each line with different symbols shows a trajectory over $112 \mathrm{~ms}$, and symbols are separated by $8 \mathrm{~ms}$. of the undulating rim tips. We found that the rim trajectories solving Eq. (11), including centrifugal force, drag, surface tension, and gravity, are quite close to experimental trajectories. However, the parabolic trajectories $\left[R(t)=d R /\left.d t\right|_{t=0} t\right.$ and $\left.Z(t)=\frac{1}{2} g t^{2}\right]$, considering only gravity, exhibit longer travel distances than the other two due to the lack of drag and surface tension and rapid downward trajectories due to the lack of centrifugal force.

The numerical result solving Eq. (11) still exhibits a slight mismatch with experiments, especially at later times. One possible explanation is that our assumption of a smooth cylindrical rim is not valid at later times due to the capillary force causing the straight rim to undulate and further turn into drops. This drop formation due to the capillary action will be discussed in the next section.

To remark on the water-glycerol cases, experiments with a water-glycerol mixture show a very short fluid sheet lifetime, mainly due to high surface tension [see Fig. 3(d)]. Therefore, no water-glycerol data is presented in this rim-dynamics section.

\section{Unstable rim: Final stage}

The traditional Plateau-Rayleigh instability [30] shows that a cylindrical jet becomes unstable and breaks into droplets. In our case, although the rim is shaped like a torus, it can be approximated as a straight cylinder because the radius of the liquid sheet is much larger than the radius of the rim (about 100 times bigger). We also assume that the attached liquid sheet does not affect the instability of the rim.

In this falling cylindrical fluid rim, we evaluate three time scales to find dominant forces for the drop formation at later times in the frame moving with the rim. First, the capillary break-up time $\left(t_{\text {capillary }}\right)$ scales as $\sqrt{\rho a^{3} / \gamma} \approx 20 \mathrm{~ms}$ where the measured rim radius $(a \approx 2 \mathrm{~mm})$ is used. The next is the extension time, which scales as $R /(d R / d t) \approx 0.1 \sim$ $1 \mathrm{~s}$ measured in experiments. Last, the viscous time scale $\left(a^{2} / v=40 \sim 200 \mathrm{~ms}\right)$ is slightly higher than the capillary timescale and lower than the extension timescale. Therefore, the primary force controlling the instability breaking a fluid rim into drops is the capillary force, while the viscous force is a secondary cause of the instability. This viscous effect slows the dynamics of capillary instability and weakly affects the most unstable mode, as its growth rate is $(\omega)_{\text {predict }}=$ $1 /\left[t_{\text {capillary }}(2 \sqrt{2}+6 \mathrm{Oh})\right]$ and its unstable wavenumber is $(k a)_{\text {predict }}=1 / \sqrt{2+3 \sqrt{2} \mathrm{Oh}}[31,32]$.

In the experiments, we have observed apparent undulations from the smooth surface of the rim after approximately $15 \mathrm{~ms}$, which is close to the capillary time scale. As this perturbation grows in time, clear drops hanging on a fluid rim are formed after about $80 \mathrm{~ms}$. Within this time scale, the rim becomes unstable due to the capillary force; however, the rim's position is not very far from the disk perimeter. Hence, the characteristic length scale is chosen to be the disk radius for further calculations.

Capillary instability sets an initial drop spacing in the beginning of this squeezing motion on the order of the capillary timescale ( $\sim 20 \mathrm{~ms}$ ). Figure 6(a) shows the formation of both the undulating rim and drops from the top view. Locations of 


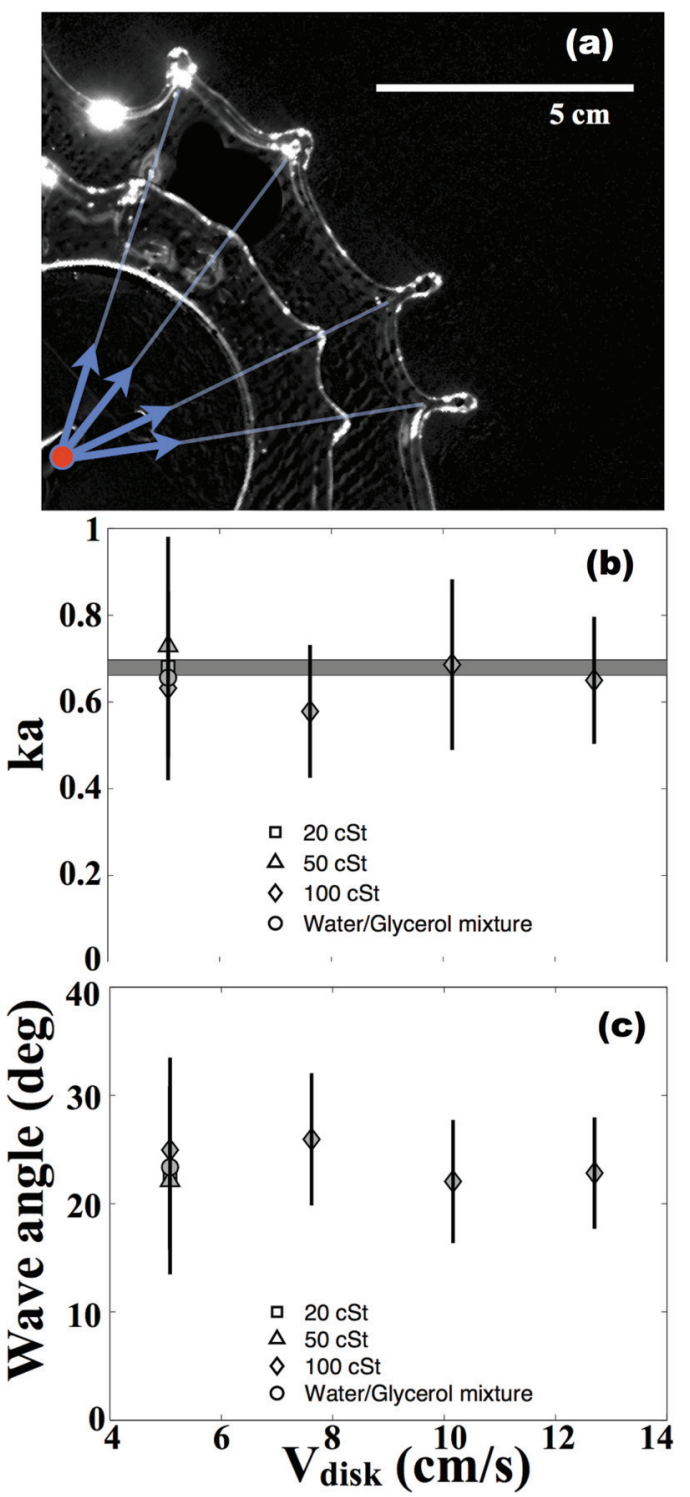

FIG. 6. (Color online) (a) Two super-imposed snapshots at 30 and $70 \mathrm{~ms}$ with viscosity of $100 \mathrm{cSt}$ and $V_{\text {plate }}=12.7 \mathrm{~cm} / \mathrm{s}$. The invariable waveangle of undulations in the course of rim expansion. Plots of (b) nondimensional wavenumber $(k a)$ and (c) waveangle ( $\phi$ in degrees) with different fluid viscosities and varying plate velocities. The gray area in (b) is the nondimensional wavenumber predicted by the Rayleigh-Plateau instability with viscous effects.

thick and protruding rim sections due to capillary force at the very beginning ( $\sim 20 \mathrm{~ms}$ ) further develop into drops as time goes on. While they fall downward and expand radially, this spacing does not change noticeably.
The Plateau-Rayleigh instability by considering viscous effects predicts $(k a)_{\text {predict }}=1 / \sqrt{2+3 \sqrt{2} \mathrm{Oh}}$, where Oh ranges from 0.02 to 0.1 for silicone-oil experiments. While our experiment changes viscosity from 20 to $100 \mathrm{cSt}$ ( $80 \%$ changes), the corresponding predicted $(k a)_{\text {predict }}$ range from 0.69 to 0.64 (only $7 \%$ changes). This result is compared with experimental measurements in Fig. 6(b). Assuming small radial expansion of the rim when capillary force determines the most unstable waveangle, the Plateau-Rayleigh instability also predicts the wave angle as $(\phi)_{\text {unstable }} \sim \frac{2 \pi a}{(k a)_{\text {predict }} R_{\text {disk }}} \sim \pi / 6.32=28.5^{\circ}$, which is close to the mean value of measurements shown in Fig. 6(c). However, some distributions in measured $k a$ and wavelangle are observed. One major source of uncertainty might come from the fluid rim interacting with a connected sheet and surrounding air. Due to small Ohnesorge numbers, the liquid rim is formed and propagates interfacial waves along the liquid sheet, and then this rim-sheet-coupled system becomes unstable [12,33], which we do not take into account in this study.

\section{CONCLUSION}

In this paper, we have studied the dynamics of a fluid squeezed by two circular disks. First, the initial spreading velocity is estimated using lubrication theory. Second, the position of a rim is described by balancing inertia with drag, centrifugal, and surface tension forces. Finally, the most unstable wavelength (or wave angle) is estimated using the traditional Rayleigh-Plateau analysis of capillary instability. Experimentally, we have tested with different clapping speeds, fluid viscosities, and surface tensions to understand the effect of fluid and kinematic properties on the dynamics and have shown that theoretically predicted rim position and drop spacing are in good agreement with experimental measurements. This study explains how sparse droplets are generated when a fluid is squeezed.

In future studies, we will continue to investigate the dependence on the diameter or shape of the plates. At the beginning of our experiment, we tested using rectangularshaped blocks and noticed that the control of initial fluid thickness on large circular or rectangular plates is rather difficult.

\section{ACKNOWLEDGMENTS}

Acknowledgment is made to the National Science Foundation (PoLS Grant No. 1205642) and the donors of American Chemical Society Petroleum Research Fund (PRF Grant No. 52332-DNI9) for support of this research. The authors thank Ian J. Jung for his contribution and for motivating this work. B.S. thanks an internship support from the Ecole Polytechnique, France.
[1] P. G. Simpkins and V. J. Kuck, J. Coll. Int. Sci. 263, 562 (2003).

[2] G. G. Nasr, A. J. Yule, and L. Bendig, Industrial Sprays and Atomization: Design, Analysis and Applications (Springer Verlag, London, 2002).
[3] P. S. Shah, L. T. Fan, I. C. Kao, and L. E. Erickson, Adv. Appl. Microbiol. 15, 367 (1972).

[4] E. Villermaux and B. Bossa, Nature Phys. 5, 697 (2009). 
[5] H. E. Edgerton, Stopping Time: The Photographs of Harold Edgerton (Abrams, New York, 1977).

[6] R. Rioboo, C. Bauthier, J. Conti, M. Voue, and J. De Coninck, Exp. Fluids 35, 648 (2003).

[7] S. T. Thoroddsen, T. G. Etoh, and K. Takehara, J. Fluid. Mech. 557, 63 (2006).

[8] Z. Levin and P. V. Hobbs, Philos. Trans. R. Soc. London A 269, 555 (1971).

[9] A. L. Yarin and D. A. Weiss, J. Fluid. Mech. 283, 141 (1995).

[10] T. T. Truscott, and A. H. Techet, J. Fluid Mech. 625, 135 (2009).

[11] G. I. Taylor, Proc. R. Soc. A. 253, 289 (1959).

[12] G. I. Taylor, Proc. R. Soc. A 253, 296 (1959).

[13] C. Clanet, J. Fluid. Mech. 430, 111 (2001).

[14] J. Aristoff, C. Lieberman, E. Chan, and J. W. M. Bush, Phys. Fluids 18, 091109 (2006).

[15] C. Clanet, Annu. Rev. Fluid Mech. 39, 469 (2007).

[16] A. M. Worthington, A Study of Splashes (Longmans, Green, and Co., London, 1908).

[17] A. M. Worthington, Philos. Trans. R. Soc. London A 189, 137 (1897).

[18] A. M. Worthington, Proc. R. Soc. London 25, 261 (1876).

[19] S. Chandra and C. T. Avedisian, Proc. Math. Phys. Sci. 432, 13 (1991).
[20] L. V. Zhang, P. Brunet, J. Eggers, and R. D. Deegan, Phys. Fluids 22, 122105 (2010)

[21] C. Josserand and S. Zaleski, Phys. Fluids 15, 1650 (2003).

[22] E. Villermaux and B. Bossa, J. Fluid Mech. 668, 412 (2011).

[23] G. Juarez, T. Gastopoulos, Y. Zhang, M. L. Siegel, and P. E. Arratia, Phys. Rev. E 85, 026319 (2012).

[24] C. Clanet and E. Villermaux, J. Fluid Mech. 462, 307 (2002).

[25] Edited by D. R. Lide, CRC Handbook of Physics and Chemistry, 72 ed. (CRC Press, Boca Raton, 1991).

[26] G. K. Batchelor, An Introduction to Fluid Dynamics (Cambridge University Press, New York, 1967).

[27] Experimental measurement with a force sensor shows the linear relation between the impact force and the disk velocity [Impact force $($ dyne $)=7.27 \times 10^{-1} V_{\text {disk }}(\mathrm{cm} / \mathrm{s})$ with $\left.R^{2}=0.736\right]$.

[28] R. B. Bird, W. E. Stewart, and E. N. Lightfoot, Transport Phenomena, 2nd ed. (John Wiley and Sons, New York, 2001).

[29] R. Clift, J. R. Grace, and M. E. Weber, Bubbles, Drops, and Particles (Dover Press, New York, 1978).

[30] L. Rayleigh, Proc. R. Soc. London 29, 71 (1879).

[31] J. Eggers and E. Villermaux, Rep. Prog. Phys. 71, 036601 (2008).

[32] C. Weber, Z. Angew. Math. Mech. 11, 136 (1931).

[33] M. Song and G. Tryggvason, Phys. Fluids 11, 2487 (1999). 